\title{
Impact of age, body weight and metabolic risk factors on steroid reference intervals in men
}

\author{
Marco Mezzullo, Guido Di Dalmazi, Alessia Fazzini, Margherita Baccini, Andrea Repaci, Alessandra Gambineri, \\ Valentina Vicennati, Carla Pelusi, Uberto Pagotto and Flaminia Fanelli \\ Unit of Endocrinology and Prevention and Care of Diabetes, Center for Applied Biomedical Research, Department of \\ Medical and Surgical Sciences, University of Bologna, S.Orsola Policlinic, Bologna, Italy \\ Correspondence \\ should be addressed \\ to F Fanelli \\ Email \\ flaminia.fanelli2@unibo.it
}

\begin{abstract}
Objective: To evaluate the independent impact of age, obesity and metabolic risk factors on 13 circulating steroid levels; to generate reference intervals for adult men.

Design: Cross-sectional study.

Methods: Three hundred and fifteen adults, drug-free and apparently healthy men underwent clinical and biochemical evaluation. Thirteen steroids were measured by LC-MS/MS and compared among men with increasing BMI. Moreover, the independent impact of age, BMI and metabolic parameters on steroid levels was estimated. Upper and lower reference limits were generated in steroid-specific reference sub-cohorts and compared with dysmetabolic subcohorts.

Results: We observed lower steroid precursors and testosterone and increase in estrone levels in men with higher $\mathrm{BMI}$ ranges. By multivariate analysis, 17-hydroxyprogesterone and dihydrotestosterone decreased with BMI, while cortisol decreased with waist circumference. Estrone increased with BMI and systolic blood pressure. Testosterone decreased with worsening insulin resistance. 17-hydroxypregnenolone and corticosterone decreased with increasing total/HDL-cholesterol ratio. Age-related reference intervals were estimated for 17-hydroxypregnenolone, DHEA, 17-hydroxyprogesterone, corticosterone, 11-deoxycortisol, cortisol and androstenedione, while age-independent reference intervals were estimated for progesterone, 11-deoxycorticosterone, testosterone, dihydrotestosterone, estrone and estradiol. Testosterone lower limit was $2.29 \mathrm{nmol} / \mathrm{L}$ lower $(P=0.007)$ in insulin resistant vs insulin sensitive men. Furthermore, the upper limits for dihydrotestosterone $(-0.34 \mathrm{nmol} / \mathrm{L}, P=0.045)$, cortisol $(-87 \mathrm{nmol} / \mathrm{L}, P=0.045-$ $0.002)$ and corticosterone $(-10.1 \mathrm{nmol} / \mathrm{L}, P=0.048-0.016)$ were lower in overweight/obese, in abdominal obese and in dyslipidaemic subjects compared to reference sub-cohorts, respectively.

Conclusions: Obesity and mild unmedicated metabolic risk factors alter the circulating steroid profile and bias the estimation of reference limits for testosterone, dihydrotestosterone, cortisol and corticosterone. Applying agedependent reference intervals is mandatory for steroid precursors and corticosteroids.
\end{abstract}

\section{Introduction}

Steroid hormone determination in humans is undergoing a profound technological upgrade, as represented by the introduction of liquid chromatography tandem mass spectrometry (LC-MS/MS) multi-analyte quantitation $(1,2)$. In the last 15 years, severe disagreements among (c) 2020 European Society of Endocrinology Printed in Great Britain immunoassays from various manufacturers and between several immunoassays and LC-MS/MS were documented, mainly on the measurement of testosterone (T), estradiol (E2), dihydrotestosterone (DHT) and cortisol (F) (3, 4, $5,6,7)$. LC-MS/MS assays based on isotopic dilution

Published by Bioscientifica Ltd. 
quantitation benefit from inherent elevated specificity and accuracy. Therefore, this technique usually provides good comparability with other LC-MS/MS assays as well as with gas chromatography-MS methods and reference procedures (2). A second major LC-MS/MS benefit consists in the possibility to evaluate challenging steroids, such as DHT and steroid metabolites as 11-deoxycortisol (11S) and corticosterone (B), whose immunometric measurement is usually not available on routine basis. Evidences on the role of these steroids in the pathogenesis, diagnosis and management of various endocrine diseases are being generated, thereby strongly supporting the extensive use of LC-MS/MS in the clinical practice $(1,8)$.

One major limitation in this path relies in the limited availability of steroid normative values. Indeed, the current knowledge about steroid circulating levels has mainly been generated by routine immunoassays (9). Therefore, an urgent need exists for updating reference intervals (RI) by means of reliable and traceable LC-MS/ MS assays. In addition, in order to achieve the utmost diagnostic efficacy, it is necessary to refine reference limits according to gender, age and pathologic conditions, such as overweight/obesity and related metabolic alterations, affecting large part of the population (10).

Male ageing has been associated with a reduction in androgen and pro-androgen secretion (11). Moreover, a mutual negative impact exists between obesity, visceral fat, insulin resistance, dyslipidaemia, hypertension and the hypothalamus-pituitary-gonadal (HPG) axis, mostly resulting in low androgen and high estrogen levels (11). Ageing and cardiovascular risk factors are also strictly related with the hypothalamus-pituitary-adrenal (HPA) axis (12). However, the modulation of adrenal steroid secretion throughout the lifespan has poorly been documented so far, as well as little is known about corticosteroid imbalance due to obesity and comorbidities.

Large heterogeneity exists in literature about the health criteria used to define the reference cohorts. Studies involving the general population often did not apply an adequate subjects' characterization by means of specialized examination and laboratory assessment. In particular, subclinical and unmedicated conditions, such as overweight, abdominal obesity, dyslipidaemia, reduced insulin sensitivity and impaired blood pressure control, are too often disregarded (9). The poor standardization of blood sampling represents a further issue, as time of the day, the nutritional status and the venepuncture stress overall affect steroid secretion to a non-negligible extent $(13,14)$.
By focusing on a cohort of adult men selected from the general population and by applying standardized sampling conditions, the present study sought to: (1.) characterize the impact of obesity and metabolic risk factors on the circulating steroid profile and (2.) provide age-specific RI for a large panel of steroids, further demonstrating how non-restrictive selection criteria could alter the estimation of reference limits.

\section{Materials and methods}

\section{Subjects}

Men aged 18-74 years were recruited from the local general population (15). The study was approved by the S.OrsolaMalpighi Hospital ethical committee (85/2008/O/Tess). All subjects signed the informed consent before being interviewed and examined by a trained endocrinologist, between $0800 \mathrm{am}$ and $1000 \mathrm{am}$ after an overnight fasting. Waist circumference and body mass index (BMI) were registered. Systolic (SBP) and diastolic (DBP) blood pressures were measured in supine position after a 3 min rest. Inclusion criteria were: BMI $\geq 18.5 \mathrm{~kg} /$ $\mathrm{m}^{2}$, weight stability in the last 3 months and complete sexual development. Steroidal and non-steroidal drug assumption in the previous 3 months (except antipyretic or non-steroidal anti-inflammatory compounds, tolerated up to 1 month before the study, and thyroxine replacement in compensated hypothyroidism), present or past endocrine, hepatic, renal, oncologic, autoimmune, cardiovascular, hematologic, neurologic or psychiatric diseases, sleep disorders, shift working, frequent flying or allergies requiring treatment caused exclusion from the study. Among 590 men examined, 213 reported assumption of one or more drugs (glucose lowering: $n=6$, cholesterol-lowering: $n=111$, anti-hypertensive: $n=83$, antidepressant: $n=13$ ), whereas, among drug-free subjects, 46 reported present or previous diseases and 10 men reported sleep disturbance. Therefore, 321 subjects were included in the present study.

\section{Biochemical and hormonal evaluation}

To avoid venepuncture stress, blood was withdrawn after 10 min saline infusion in Vacuette $Z$ serum beads clot activator tubes (Greiner Bio-One, Kremsmunster, Austria). After $20 \mathrm{~min}$ settling, tubes were centrifuged $(2000 \boldsymbol{g}$, $10 \mathrm{~min}$, room temperature) and serum for LC-MS/MS was stored at $-80^{\circ} \mathrm{C}$. Routine analyses on fresh blood 
(intra- and inter-assay CV\%) were performed: insulin (1.5 and 4.9\%), total cholesterol (<1.0 and 2.7\%), HDL cholesterol $(<0.95$ and $1.3 \%)$ and triglycerides $(<1.5$ and $1.8 \%$ ) were measured by Modular Analytics E170 (Roche Diagnostics). Glucose was measured by Breeze-2 glucometer (Bayer <4.5\%). The homeostatic model assessment-insulin resistance (HOMA-IR) and the total/ HDL-cholesterol ratio were computed $(16,17)$.

\section{Steroid measurement by LC-MS/MS}

Thirteen steroid hormones were measured by two validated in-house LC-MS/MS assays, verified by certified reference materials and by multicentre ring trials (18). The first included F, 11S, B, 11-deoxycorticosterone (DOC), progesterone (P4), 17-hydroxyprogesterone (17OHP4), dehydroepiandrosterone (DHEA), androstenedione (A4) and T (15), and the second included 17-hydroxypregnenolone (17OHP5), DHT, estrone (E1) and E2 (19). Assays main information are reported in Supplementary Table 1 (see section on supplementary materials given at the end of this article).

\section{Study design}

After the removal of six subjects showing far outlier data (two 17OHP5, three 17OHP4 and one DOC values), the cohort was stratified according to the BMI level in normal weight (NW, $18.5<\mathrm{BMI} \leq 25.0 \mathrm{~kg} / \mathrm{m}^{2} ; n=175$ ), overweight (OW, $25.0<\mathrm{BMI} \leq 30.0 ; n=106)$, class 1 obese (OB1, $30.0<\mathrm{BMI} \leq 35.0 ; n=24)$ and class 2 plus class 3 obese (OB2, $35.0<\mathrm{BMI} \leq 45.0 ; n=10)$. Anthropometric and metabolic features as well as steroid levels were compared among those BMI classes. Afterward, we assessed the independent impact of age, BMI, waist circumference, SBP, DBP, HOMA-IR, total/HDL-cholesterol and triglycerides on each steroid by multiple regression. Steroids were therefore classified in four sub-panels, as their levels resulted impacted by (1.) ageing, (2.) age plus one or more metabolic parameters, (3.) one or more metabolic parameters but not age or (4.) by none of the tested parameters. RI were defined as the central 95\% of hormone distribution. For sub-panels 1 and 4, lower (LRL) and upper (URL) reference limits were estimated in the whole cohort. For sub-panels 2 and 3, limits were estimated in reference sub-cohorts of subjects displaying normal values of the parameter(s) identified for influencing the particular steroid levels according to the multiple regression result. To this aim, we identified subjects with normal $(\leq 102 \mathrm{~cm}$, norWC; $n=268)$ or elevated (>102 cm, dysWC; $n=47$ ) waist circumference, with normal $(<2.5$, norHOMA-IR; $n=132)$ or altered ( $\geq 2.5$, dysHOMA-IR; $n=35)$ HOMA-IR and with normal $(<5$, norChol; $n=146)$ or altered $(\geq 5$, dysChol; $n=46)$ total/HDL-cholesterol. Additionally, we defined a sub-cohort of NW subjects having normal SBP $(\leq 130 \mathrm{mmHg}$ ) (NW and norSBP; $n=63$ ) and a sub-cohort of subjects who were OW and/or had altered SBP $(\geq 145$ mmHg) (OW and/or dysSBP; $n=150$ ).

Age-specific RI were estimated for steroids in subpanels 1 and 2. Unique age-independent RI were estimated for steroids in sub-panels 3 and 4. Finally, to evaluate whether alterations in metabolic parameters influencing the steroid values could also influence the RI estimation, LRL and URL estimated in the reference sub-cohorts were compared with lower and upper limits calculated in subcohorts displaying the defined dysmetabolic features, respectively.

\section{Statistical analysis}

Source variables were not normally distributed at the Kolmogorov-Smirnov test and significantly skewed, therefore, Box Cox transformation was applied (20). Far outliers data were detected by the Tukey's test (21). Descriptive and steroid variables were compared among classes with increasing BMI range by the ANOVA trend test available on IBM SPSS package v. 20, IBM Co. Comparison between pairs of BMI classes was performed by Tuckey's HSD method for linear contrast analysis. Homogeneity of variance was not assumed for variables showing significant $P$ value at the Levene's test. The stepwise multiple regression included age, BMI, waist circumference, SBP, DBP, HOMA-IR, total/HDL-cholesterol and triglyceride levels as covariates and each steroid as the dependent variable. The effect size $\left(f^{2}\right)$ was estimated by Cohen's method for multiple regression according to the formula: $f^{2}=\frac{s r^{2}}{\left(1-R^{2} \text { full }\right)}$, where $s r$ is the semipartial correlation coefficient for the predictor of interest and $R$ is the full correlation coefficient of the multiple regression model (22). LRL and URL were estimated as the mean $-1.96 \times$ s.D. and mean $+1.96 \times$ s.D. of the transformed variables, respectively, and the values were back-transformed to the original unit (23). As 88 and $23 \%$ of the subjects displayed DOC and P4 levels below the LC-MS/MS sensitivity limits (0.236 and $0.156 \mathrm{nmol} / \mathrm{L}$, respectively), respectively, their distribution could not be normalized. Therefore, the comparison of these steroids among BMI classes was performed by Kruskal-Wallis analysis, the URL was calculated as the 97.5 centile of 
distribution and multiple stepwise regression was not performed.

Age-specific RI were estimated by modeling the transformed steroid variable on age distribution, according to the fractional polynomial regression by Royston and Wright (24). Age $(X)$ was transformed in order to stabilize the steroid variable $(Y)$ for large values of $X$ according to the formula: $e X=\exp \left(\frac{(\log (0.01) \times(X-\min (X)))}{(\max (X)-\min (X))}\right)$.

Then, the optimal model was selected for providing the lowest polynomial degree (parsimony) with maximum decrease in deviance (goodness of fit). Best-fit polynomial coefficients were selected by fp syntax in STATA version 13.0 (StataCorp LLC). RI were visually inspected using xrigls syntax in STATA.

Lower and upper limits were compared between reference and dysmetabolic sub-cohorts by the $z$ distribution. Two-tailed $P$ values $<0.05$ were considered significant. Data were analysed by MedCalc v.18.2.1 (MedCalc Software, Mariakerke, Belgium) except when specified.

\section{Results}

\section{Anthropometric, metabolic and hormonal features of the cohort}

The final cohort included 315 subjects. Table 1 reports the descriptive and hormonal features observed in NW $(n=175)$, OW $(n=106)$, OB1 $(n=24)$ and OB2 $(n=10)$ men. Men in classes at increasing BMI displayed increasing age $(P=0.042)$, waist circumference, SBP, DBP, insulin, HOMA-IR, total/HDL-cholesterol, triglycerides (all $P<0.001)$, glucose $(P=0.007)$ and total cholesterol $(P=0.003)$ levels, as well as lowering HDL levels $(P<0.001)$.

Among steroid hormones, we observed a significant decrease in 17OHP5 $(P=0.029)$, P4,d T (both $P<0.001)$ and 17OHP4 $(P=0.001)$ and a significant increase in $\mathrm{E} 1$ $(P<0.001)$ in classes at increasing BMI. In particular, paired comparisons revealed that, with respect to NW, OW showed lower P4 $(P=0.002), \mathrm{OB} 1$ showed lower P4 $(P=0.009)$ and $\mathrm{T}(P<0.001)$, whereas OB2 displayed lower P4 $(P=0.010), 17$ OHP4 $(P=0.015)$ and $\mathrm{T}(P<0.001)$. OB1 and OB2 also showed lower T levels compared to OW (both $P<0.001)$. In addition, E1 levels in OB2 were significantly higher compared to NW $(P=0.002)$ and OW $(P=0.030)$. Though the trend analysis among BMI classes did not achieve statistical significance $(P=0.060)$, we found lower DHT levels in OB1 compared with NW $(P=0.004)$.

\section{Independent impact of age, anthropometric and metabolic parameters on steroid levels}

Results from the multiple stepwise regression are shown in Table 2 and Supplementary Fig. 1. Age associated with decreasing 17OHP5, DHEA, B and A4 (all $P<0.001$ ), 17OHP4 $(P=0.007), 11 \mathrm{~S}$ and $\mathrm{F}$ (both $P=0.010)$, but not with T, DHT, E1 and E2 levels. A large effect size was observed for DHEA $\left(f^{2}=0.86\right)$ and 17OHP5 $\left(f^{2}=0.51\right)$, whereas for other steroids it accounted for less than 0.20. BMI negatively influenced 17OHP4 $\left(f^{2}=0.06\right.$, $P=0.003)$ and DHT $\left(f^{2}=0.06, P=0.005\right)$, while positively affecting E1 $\left(f^{2}=0.05, P=0.007\right)$. E1 also directly associated with SBP $\left(f^{2}=0.03, P=0.046\right)$. F decreased with increasing waist circumference $\left(f^{2}=0.05, P=0.005\right)$, while $\mathrm{T}$ decreased with increasing HOMA-IR $\left(f^{2}=0.11\right.$, $P<0.001)$. Total/HDL-cholesterol inversely associated with 17OHP5 $\left(f^{2}=0.04, P=0.039\right)$ and B $\left(f^{2}=0.09\right.$, $P<0.001)$. No steroid associations were observed with DBP and triglycerides.

According to these results, DHEA, A4 and 11S were classified in sub-panel 1 (only influenced by age), 17OHP5, 17OHP4, B and F in sub-panel 2 (influenced by age plus metabolic parameters), T, DHT and E1 in sub-panel 3 (influenced by metabolic parameters) and E2 in sub-panel 4 (not influenced by the tested parameters).

\section{Steroid reference intervals}

Age-specific RI were generated within the whole cohort $(n=315)$ for DHEA, 11 S and A4 (sub-panel 1), within NW $(n=175)$ for $17 \mathrm{OHP} 4$, within norWC $(n=268)$ for $\mathrm{F}$ and within norChol men $(n=146)$ for 17 OHP5 and B (subpanel 2) (Fig. 1). The largest decline from age 20 to 60 years in LRL and URL was displayed by DHEA (-68.9 and $-71.0 \%$, respectively) and 17OHP5 (-61.9 and $-72.5 \%$, respectively), followed by B ( -38.4 and $-47.5 \%$, respectively) and $\mathrm{A} 4$ ( -23.0 and $-42.5 \%$, respectively). Moreover, 17OHP4, 11S and F showed an URL decline of $-29.0 \%,-25.0 \%$ and $-18.9 \%$, respectively. Detailed RI at every fifth year are listed in Table 3.

Age-independent RI were generated in the reference sub-cohort including norHOMA-IR $(n=132)$ for T, NW $(n=175)$ for DHT, NW and norSBP men $(n=63)$ for E1 (all sub-panel 3) and in the whole cohort $(n=315)$ for E2 (sub-panel 4) (Table 4). DOC and P4 URL calculated in the whole cohort $(n=315)$ were 0.292 and $0.493 \mathrm{nmol} / \mathrm{L}$, respectively. 
Table 1 Anthropometric, metabolic and hormonal features of the cohort.

\begin{tabular}{|c|c|c|c|c|c|}
\hline & $\begin{array}{l}\text { Normal weight } \\
(\mathrm{NW}, n=175)\end{array}$ & $\begin{array}{l}\text { Overweight } \\
\text { (OW, } n=106)\end{array}$ & $\begin{array}{l}\text { Class } 1 \text { Obese } \\
(\mathrm{OB} 1, n=24)\end{array}$ & $\begin{array}{l}\text { Class } 2 \text { and } 3 \text { Obese } \\
\qquad(\mathrm{OB} 2, n=10)\end{array}$ & $\begin{array}{c}\text { ANOVA } \\
\text { trend } P \\
\text { value }\end{array}$ \\
\hline \multicolumn{6}{|l|}{ Age, years } \\
\hline $\begin{array}{l}\text { Mean } \pm \text { S.D. } \\
\text { Min-max }\end{array}$ & $40.4 \pm 13.7$ & $49.0 \pm 12.1^{\mathrm{a}}$ & $50.8 \pm 12.6^{b}$ & $48.7 \pm 11.5$ & 0.042 \\
\hline \multicolumn{6}{|c|}{ Body mass index, $\mathrm{kg} / \mathrm{m}^{2}$} \\
\hline Mean \pm S.D. & $22.7 \pm 1.5$ & $26.6 \pm 1.4^{\mathrm{a}}$ & $31.7 \pm 1.5^{c, d}$ & $38.6 \pm 2.7^{e, f, g}$ & $<0.001$ \\
\hline Min-max & $18.6-24.9$ & $25.0-29.9$ & $30.0-34.8$ & $35.1-44.2$ & \\
\hline \multicolumn{6}{|c|}{ Waist circumference, $\mathrm{cm}$} \\
\hline Mean \pm S.D. & $83.8 \pm 7.0$ & $95.6 \pm 6.8^{a}$ & $109.3 \pm 5.6^{c, d}$ & $123.1 \pm 7.8^{\mathrm{e}, \mathrm{f}, \mathrm{g}}$ & $<0.001$ \\
\hline Min-max & 66-99 & 80-115 & 100-119 & 114-135 & \\
\hline \multicolumn{6}{|c|}{ Systolic blood pressure, $\mathrm{mmHg}$} \\
\hline Mean \pm S.D. & $124.1 \pm 15.4$ & $130.2 \pm 15.9^{h}$ & $143.3 \pm 17.9^{c, i}$ & $143.1 \pm 17.9$ & $<0.001$ \\
\hline Min-max & $100-180$ & 105-190 & $120-180$ & 110-170 & \\
\hline \multicolumn{6}{|c|}{ Diastolic blood pressure, $\mathrm{mmHg}$} \\
\hline Mean \pm S.D. & $81.3 \pm 7.6$ & $85.4 \pm 7.9^{1}$ & $89.0 \pm 10.2^{c}$ & $92.0 \pm 10.3^{m}$ & $<0.001$ \\
\hline Min-max & $65-100$ & 65-105 & $70-110$ & 80-110 & \\
\hline \multicolumn{6}{|l|}{ Glucose, mg/dL } \\
\hline Mean \pm S.D. & $81.1 \pm 10.7$ & $86.2 \pm 13.0^{\prime}$ & $90.9 \pm 15.8^{b}$ & $91.8 \pm 16.1$ & 0.007 \\
\hline Min-max & $52-107$ & 56-126 & 61-123 & $71-126$ & \\
\hline \multicolumn{6}{|l|}{ Insulin, $\mu \mathrm{U} / \mathrm{mL}$} \\
\hline Mean \pm S.D. & $5.2 \pm 2.9$ & $7.8 \pm 4.0^{a}$ & $12.0 \pm 5.5^{c, d}$ & $21.6 \pm 5.6^{n}$ & $<0.001$ \\
\hline Min-max & $1.6-21.8$ & $2.6-23.0$ & 6.0-29.6 & $11.0-33.5$ & \\
\hline \multicolumn{6}{|l|}{ HOMA-IR } \\
\hline Mean \pm S.D. & $1.14 \pm 0.63$ & $1.66 \pm 1.03^{a}$ & $2.56 \pm 1.32^{c, i}$ & $4.78 \pm 1.79^{e, f, n}$ & $<0.001$ \\
\hline Min-max & $0.33-4.58$ & $0.60-6.08$ & $1.22-7.02$ & 2.44-7.69 & \\
\hline \multicolumn{6}{|c|}{ Total cholesterol, mg/dL } \\
\hline Mean \pm S.D. & $180.3 \pm 37.5$ & $199.8 \pm 31.8^{a}$ & $203.1 \pm 48.0$ & $210.6 \pm 22.7^{\circ}$ & 0.003 \\
\hline Min-max & $99-270$ & $107-320$ & $123-305$ & $182-243$ & \\
\hline \multicolumn{6}{|l|}{$\mathrm{HDL}, \mathrm{mg} / \mathrm{dL}$} \\
\hline Mean \pm S.D. & $52.8 \pm 13.2$ & $48.3 \pm 11.2$ & $41.6 \pm 10.3^{c, p}$ & $39.8 \pm 4.0^{m}$ & $<0.001$ \\
\hline Min-max & 33-96 & 23-88 & $27-70$ & $35-48$ & \\
\hline \multicolumn{6}{|c|}{ Total/HDL-cholesterol ratio } \\
\hline Mean \pm S.D. & $3.47 \pm 0.98$ & $4.21 \pm 1.20^{a}$ & $4.77 \pm 1.44^{c}$ & $5.27 \pm 0.66^{e}$ & $<0.001$ \\
\hline Min-max & $1.72-6.62$ & $1.75-8.30$ & $2.82-8.03$ & $4.67-6.75$ & \\
\hline \multicolumn{6}{|c|}{ Triglycerides, mg/dL } \\
\hline Mean \pm S.D. & $69.5 \pm 38.3$ & $94.2 \pm 60.8^{a}$ & $121.6 \pm 53.3^{c}$ & $143.3 \pm 64.3^{e}$ & $<0.001$ \\
\hline Min-max & $16-274$ & 48-379 & $52-251$ & 94-287 & \\
\hline \multicolumn{6}{|c|}{ 17Hydroxypregnenolone (17OHP5), nmol/L } \\
\hline Mean \pm S.D. & $8.75 \pm 7.46$ & $7.39 \pm 6.51$ & $6.87 \pm 5.92$ & $5.53 \pm 2.86$ & 0.029 \\
\hline Min-max & $2.06-39.79$ & $1.68-42.46$ & $1.93-24.22$ & $2.66-10.80$ & \\
\hline \multicolumn{6}{|l|}{ DHEA, nmol/L } \\
\hline Mean \pm S.D. & $16.7 \pm 12.5$ & $13.9 \pm 11.0$ & $14.0 \pm 15.3$ & $13.3 \pm 6.1$ & 0.141 \\
\hline Min-max & $3.4-77.2$ & $2.7-63.1$ & $4.4-60.3$ & $6.9-29.0$ & \\
\hline \multicolumn{6}{|c|}{ Progesterone (P4), nmol/L } \\
\hline Median (IQR) & $0.251(0.177-0.318)$ & $0.211(0.156-0.267)^{1}$ & $0.200(0.156-0.235)^{b}$ & $0.156(0.156-0.242)^{\mathrm{m}}$ & $<0.001^{\#}$ \\
\hline Min-max & $0.156-0.665$ & $0.156-0.528$ & $0.156-0.436$ & $0.156-0.270$ & \\
\hline \multicolumn{6}{|c|}{ 17Hydroxyprogesterone (17OHP4), nmol/L } \\
\hline Mean \pm S.D. & $3.34 \pm 1.33$ & $3.09 \pm 1.15$ & $2.73 \pm 1.30$ & $2.27 \pm 0.84^{\circ}$ & 0.001 \\
\hline Min-max & $0.94-7.19$ & $1.20-6.86$ & $0.82-6.41$ & $1.24-4.17$ & \\
\hline \multicolumn{6}{|c|}{ Corticosterone (B), nmol/L } \\
\hline Mean \pm S.D. & $9.89 \pm 8.88$ & $9.63 \pm 8.16$ & $10.53 \pm 8.24$ & $6.99 \pm 2.97$ & 0.180 \\
\hline Min-max & $1.20-49.39$ & $2.25-45.81$ & $2.11-37.41$ & $3.15-11.95$ & \\
\hline \multicolumn{6}{|c|}{ 11Deoxycorticosterone (DOC), $\mathrm{nmol} / \mathrm{L}$} \\
\hline Median (IQR) & $0.236(0.236-0.236)$ & $0.236(0.236-0.236)$ & $0.236(0.236-0.236)$ & $0.236(0.236-0.236)$ & $0.330^{\#}$ \\
\hline Min-max & $0.236-0.443$ & $0.236-0.265$ & $0.236-0.236$ & $0.236-0.236$ & \\
\hline 11Deoxycortisol & & & & & \\
\hline Mean \pm S.D. & $0.978 \pm 0.732$ & $0.975 \pm 0.731$ & $1.205 \pm 0.927$ & $0.945 \pm 0.528$ & 0.855 \\
\hline Min-max & $0.225-4.312$ & $0.225-3.764$ & $0.263-4.130$ & $0.358-1.804$ & \\
\hline
\end{tabular}


Table 1 Continued.

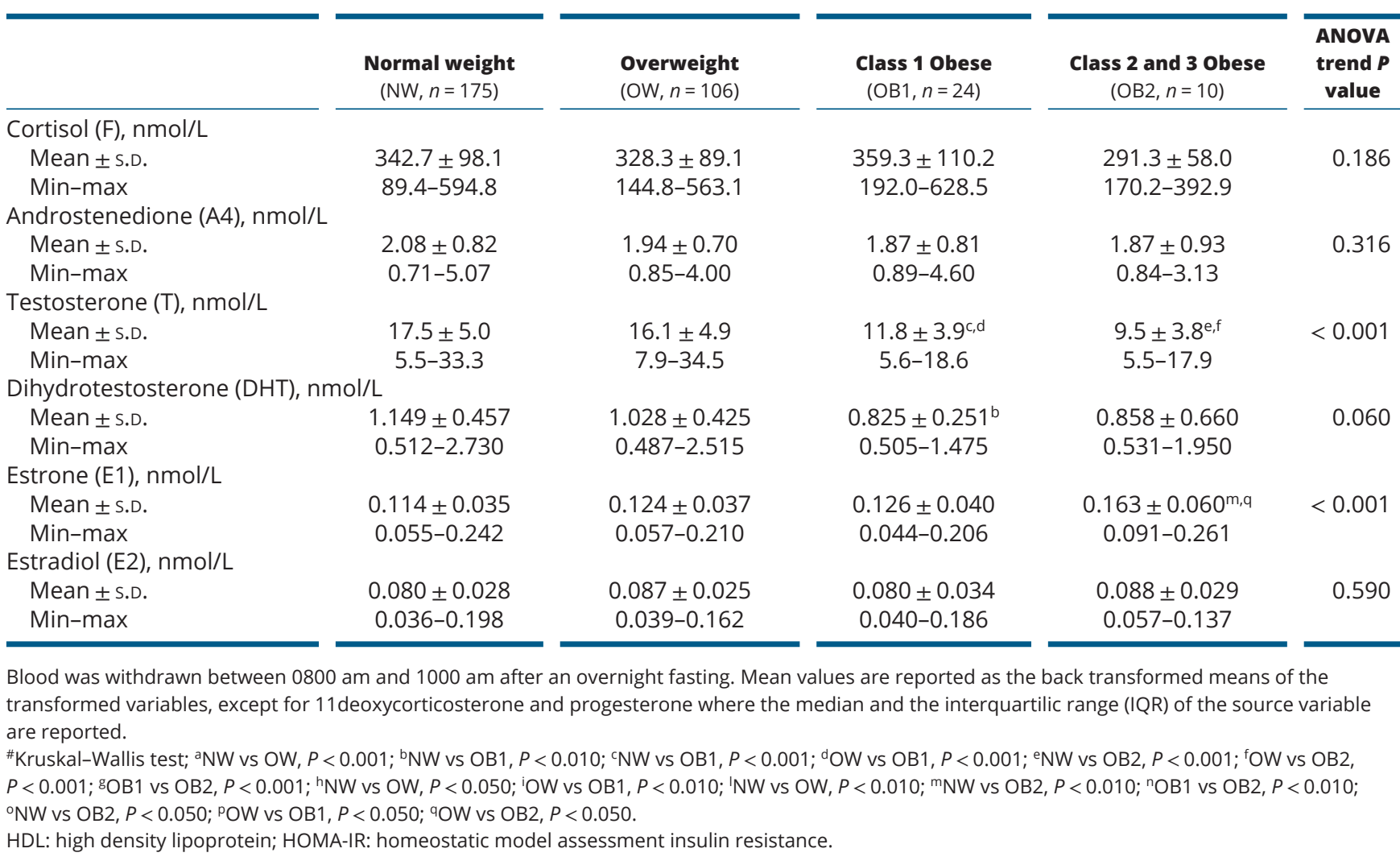

Impact of metabolic risk factors on the estimation of reference limits

LRL and URL from the reference sub-cohorts were compared with lower and upper limits calculated in $\mathrm{OW}$ $(n=140)$ for $17 \mathrm{OHP} 4$ and DHT, in dysWC $(n=47)$ for $\mathrm{F}$, in dysChol $(n=46)$ for 17 OHP5 and B, in dysHOMA-IR $(n=35)$ for $\mathrm{T}$ and in OW and/or dysSBP $(n=150)$ subjects for E1, respectively. For 17OHP5, 17OHP4, B and F, comparisons were performed at every year of age, whereas for T, DHT and E1, the age-independent limits were compared (Fig. 2 and Table 4).

Upper limits for B in dysChol at ages 45-59 years were significantly lower compared with URL in norChol, with differences ranging from -9.6 to $-10.4 \mathrm{nmol} / \mathrm{L}$ (average -10.1 nmol/L, -30.7\%, P: 0.016-0.048). Moreover, F upper limits in dysWC at ages 46-65 years were significantly lower compared to URL in norWC, with differences ranging from -45 to $127 \mathrm{nmol} / \mathrm{L}$ (average $-87 \mathrm{nmol} / \mathrm{L}$, $-17.6 \%, P$ : 0.002-0.045). No differences were detected in $17 \mathrm{OHP} 5$ and 17OHP4 limits between the reference and dysmetabolic sub-cohorts (Fig. 2).

$\mathrm{T}$ lower limit estimated in dysHOMA-IR was significantly lower than the LRL found in norHOMA-IR
(-2.29 nmol/L, -26.9\%, $P=0.007)$; however, significance was not achieved for the upper limit. At variance, DHT upper limit in OW was significantly lower than the URL calculated in NW $(-0.34 \mathrm{nmol} / \mathrm{L},-14.5 \%, P=0.045)$; however, significance was not achieved for the lower limit. E1 limits calculated in the sub-cohorts of OW and/ or dysSBP subjects were not different from reference limits in NW and norSBP sub-cohort (Table 4).

\section{Discussion}

The current knowledge about steroid circulating levels has predominantly been generated by immunoassays, often criticized for lacking accuracy, sensitivity and reproducibility. Moreover, steroid precursors and metabolites not measured on a routine basis have largely been neglected (25). Besides improving the quality of the measurement, LC-MS/MS enlarges the panel of measurable steroids, thereby prompting its application in several research areas $(1,10)$. Therefore, there is a need to re-define normal values and major confounding factors influencing the multitude of steroids now easily measurable in the bloodstream. 


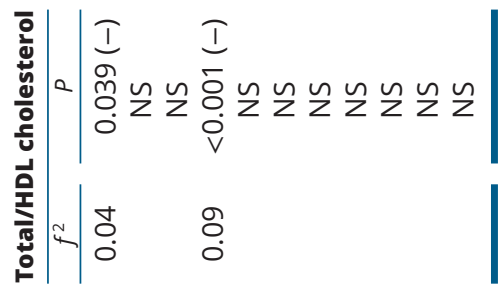

To limit the influence of confounding factors, our subjects selected from the general population were visited by a trained endocrinologist to exclude those affected by any disease or taking drugs. Moreover, we standardized procedural aspects such as the fasting condition, the early morning withdrawal and the avoidance of venepuncture stress. By monitoring easily accessible anthropometric and metabolic parameters, we found that more than half of our apparently healthy individuals displayed variable degrees of adiposity, hypertension, insulin resistance and dyslipidaemia. In the present study, we therefore provide novel data on the impact of excess weight, metabolic impairment and ageing on 13 circulating steroids.

In our cohort, the increasing severity of the obese status was paralleled by a linear decrease in delta- 4 and delta-5 steroid precursors and by an imbalance in the
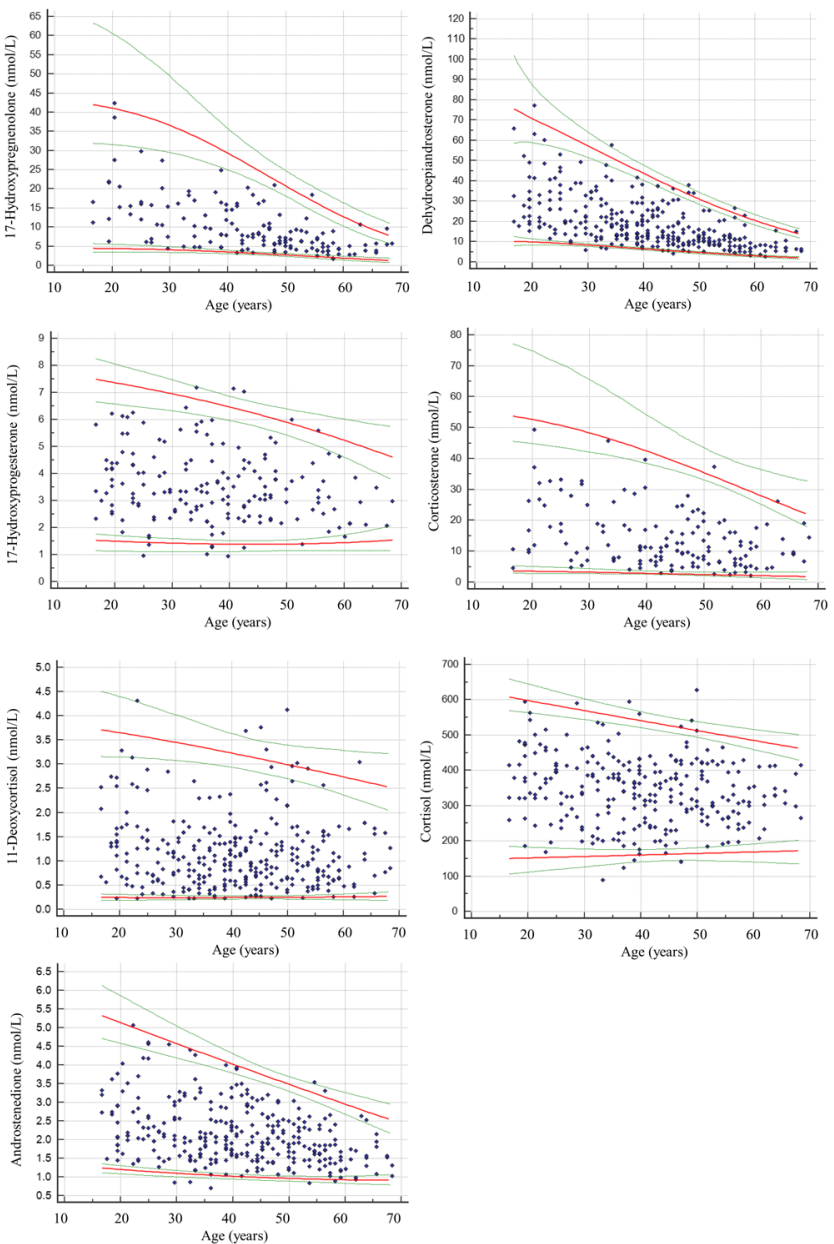

Figure 1

Distribution of steroid hormone levels in serum by age in the reference sub-cohorts. Continuous lines: lower and upper reference limits; dashed lines: 90\% confidence intervals. 
sex steroids T, DHT and E1. Besides, corticosteroids, pro-androgens and E2 levels were unvaried. As excess weight and ageing are most often clustering with insulin resistance, dyslipidaemia and hypertension, we tried to dissect which specific alteration was independently influencing steroid levels. By this approach, we found that ageing has no independent effect on T, DHT, E1 and E2 levels. Conversely, BMI is independently related with lowering DHT and increasing E1. Such a finding is in line with the known androgen/estrogen imbalance resulting by the increased pro-androgens aromatization and by the elevated $3 \alpha$-reduction of DHT in the hypertrophic adipose tissue (26). Among the several studies focused on the relationship between ageing, obesity, metabolic alterations and the male HPG, only a few used MS-based steroid assays. Recently, Travison et al. showed a 30\% decrease in T LRL from age 40 to 80 years in non-obese men, also reporting that, when obese individuals where included in the reference population, the LRL was further reduced down to 53\% (27). In another study in which 40-80 years old men were reporting very good or excellent health, obesity had an age-independent relationship with lowering $\mathrm{T}$ and DHT and increasing E1 and E2 (28). In the same study (28) and in others (29, $30)$, sex steroids were not or only minimally associated with ageing. Finally, Kelsey et al. derived from multiple studies that $T$ levels peak around age 19 years and that no decline occurred after age 40 years (31). Taken together, these data suggest that age-related changes in sex steroids may rather be due to comorbidities accumulating with ageing than to ageing per se $(28,29,30,31)$. In partial agreement with these findings, we found HOMA-IR as the sole independent determinant of $\mathrm{T}$ levels. However, our study lacks sex hormone binding globulin and free $\mathrm{T}$ measurements, which could have helped in better characterizing the apparent hypotestosteronemia in men with reduced insulin sensitivity. In line with recent data (32), E2 levels in our cohort were not influenced by any of the tested parameters.

At variance from sex steroids, in our hands, steroid precursors and corticosteroids showed a steep decrease with ageing. The extent of the decrease was particularly relevant for DHEA and 17OHP5, and moderate for A4 and $\mathrm{B}$, overall showing a reduction from age 20 to 60 years ranging between 20 and 70\%. A 30\% decline or less was also detected in 17OHP4, 11S and F URL across the same age range. Interestingly, when adjusting for age and metabolic parameters, the effect of BMI was only maintained on 17OHP4. As 17OHP4 is a crucial crossway precursor for both the gonadal and the adrenal steroidogenesis, we 


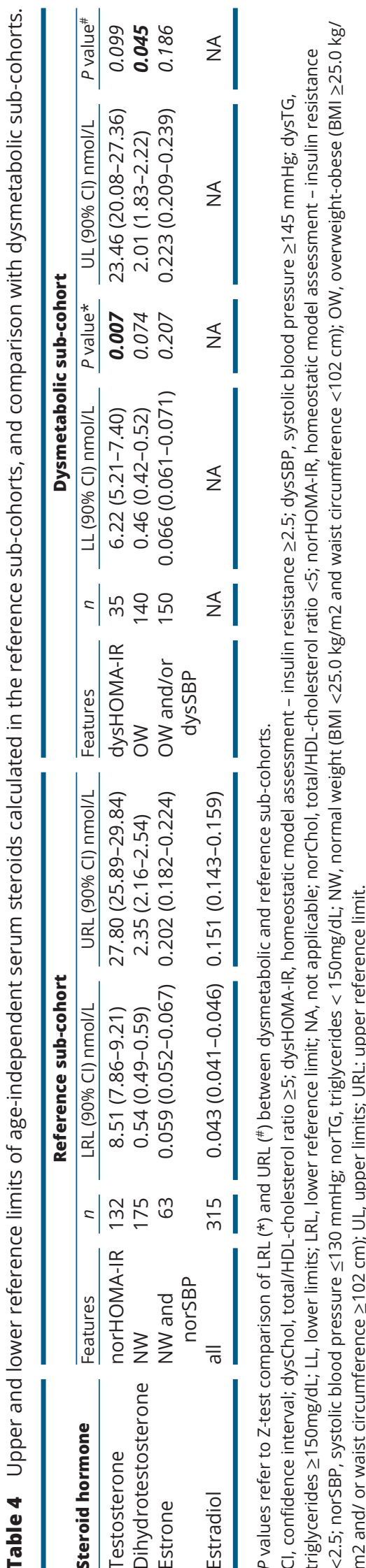

cannot postulate whether this association affects the HPA or the HPG. Furthermore, we showed an inverse association between $\mathrm{F}$ and abdominal obesity. Such a finding is in apparent contrast with the known mutual
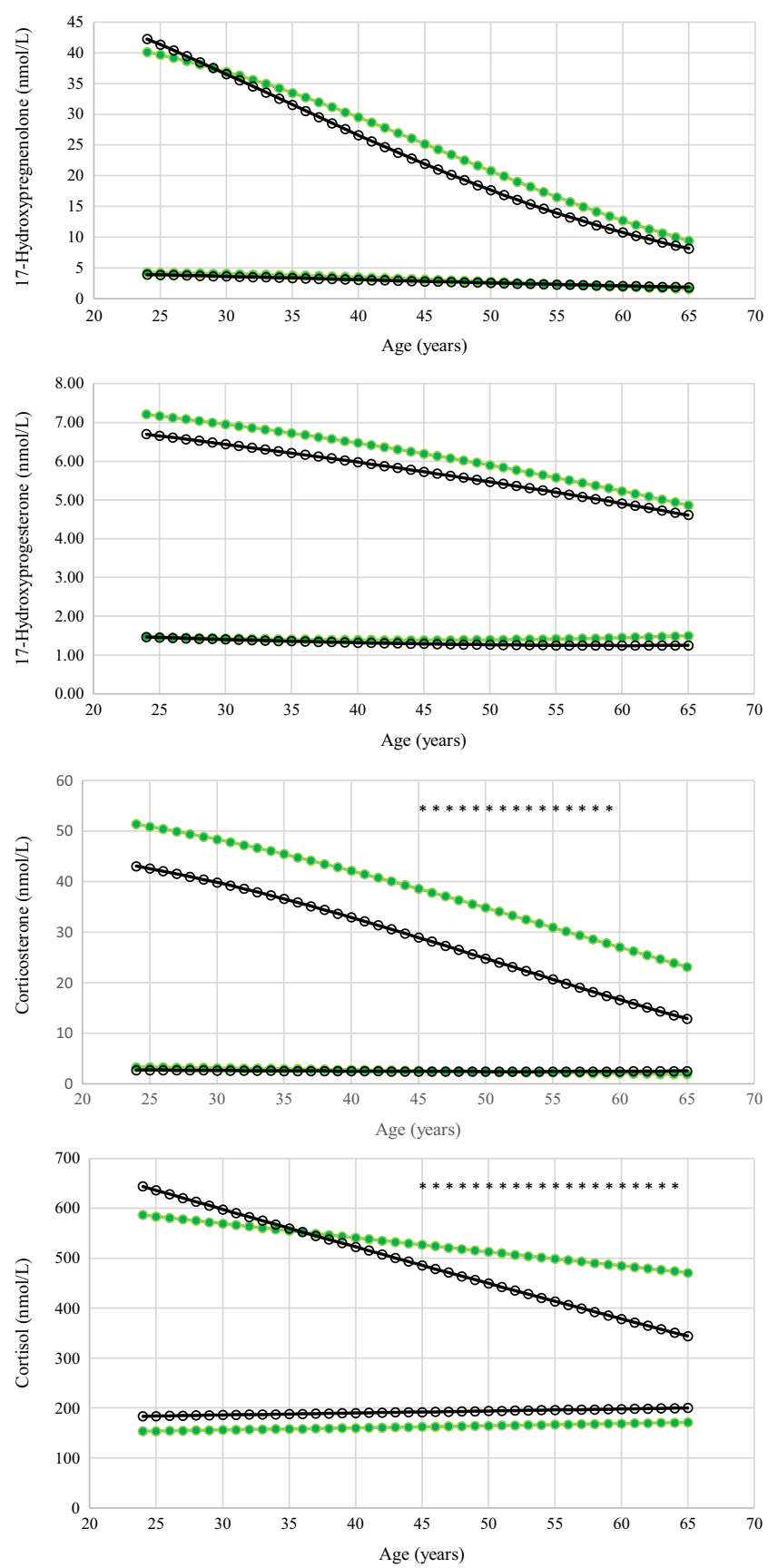

Figure 2

Steroid lower and upper limits per year of age in reference and dysmetabolic sub-cohorts. Black lines and white dots: lower and upper limits calculated in the reference sub-cohorts. Green lines and dots: lower and upper limits calculated in the dysmetabolic sub-cohorts. ${ }^{*} P<0.05$. 
association of chronic hypercortisolism with visceral fat accumulation and metabolic impairment. Nonetheless, in agreement with our finding, low early morning $\mathrm{F}$ levels were previously described in obese individuals, and the paradox was explained by the loss of HPA circadian rhythmicity, inducing low F secretion in the morning and high in the evening, and by the increased F clearance (33, 34). At variance from previous studies, we did not observe any association between $\mathrm{F}$ and adverse metabolic features, possibly because our cohort did not overall exhibit severe metabolic impairments (33).

We do not have a clear explanation for the inverse relationship of the total/HDL-cholesterol ratio with 17OHP5 and B. However, according to Cherradi and coauthors, HDL represents a major source for angiotensin II-mediated mineralocorticoid biosynthesis in adrenal cells, as assessed by measuring aldosterone and pregnenolone (35). We can therefore speculate that the positive association of 17OHP5, the direct product of pregnenolone, and $\mathrm{B}$, the direct precursor of aldosterone, with HDL relative abundance in the cholesterol pool, could possibly be dependent on biochemical rather than dysmetabolic mechanisms.

No full consensus exists among the few recent studies analysing the impact of ageing, obesity and comorbidities on large circulating LC-MS/MS steroid profiles. Similar to our results, Eisenhofer et al. described an age decline in men from 22 to 70 years for DHEA, 17OHP4, B, 11S, $\mathrm{F}$ and $\mathrm{A} 4$ and an inverse association between $\mathrm{BMI}$ and P4, 17OHP4, DOC, B, F and T, but not $11 \mathrm{~S}$ and A4. They also found an inverse association between hypertension and 17OHP4; however, they did not evaluate the effect of insulin resistance and dyslipidemia (36). DamgaardOlesen and co-authors reported an age decline in men aged 30-60 years in 17OHP5, DHEA and A4, but not in 17OHP4 levels. They also described the negative impact of BMI on DHEA, 17OHP4, A4 and T and of metabolic syndrome on DHEA, 17OHP4 and T; however, relationships between steroids and individual metabolic parameters were not reported (37). Two other recent studies confirmed the agedecline in 17OHP5, DHEA and A4 in men of similar age $(29,30)$.

Analysing steroid relationships with various comorbidities allowed us to define specific reference sub-cohorts with the largest possible sample size, thus ensuring both unbiased and robust RI estimation. We also showed that, when calculated in dysmetabolic sub-cohorts, T, DHT, F and B limits were significantly different from reference limits, thereby demonstrating that tolerating reduced insulin sensitivity, overweight/ obesity, excess visceral fat and dyslipidaemia may result in biased reference limit estimation. Hence, our study suggests that even mild metabolic imbalances are able to affect steroid secretion and metabolism, resulting in an altered circulating profile.

As reported in our recent review (9), consistent disagreements are found among RI reported so far, which are more severe for steroids usually not measured in the clinical routine $(27,29,30,36,37,38,39,40,41)$. Reasons for such a disagreement in steroid levels as well as in their association with anthropometric and metabolic parameters could be found at different levels. As our data demonstrate, the clinical and biochemical characterization of the reference cohort influences steroid values. The metabolic parameters that we monitored are commonly available in routine clinical laboratories and should therefore be always considered. Most of the previous studies apparently did not exclude subjects taking drugs, some of them excluding steroidal drugs $(29,30,36,37,38,39,40,41)$. The exact time of blood withdrawal and the fasting status, both of which are known to impact steroid levels, are often not specified, whereas measures to avoid venepuncture stress were apparently not taken (9). The impact of the latter on cortisol secretion was repeatedly reported in literature (42). Conversely, to the best of our knowledge, no data are available on the impact of venepuncture stress on glucocorticoid metabolites and on androgen precursors. Such an aspect should therefore be investigated as a potential non-negligible confounding factor undermining the interpretation of information generated by novel large steroid-profiling techniques and their efficacy in clinical settings. In addition, the multivariate design may influence the associative observations. As to the elaboration of age-dependent data, our approach provided a continuous age modeling of RI based on a reasonable number of subjects, thus obviating data partitioning into arbitrary age categories $(13,23)$. Finally, even though LC-MS/MS is supposed to guarantee high accuracy, analytical performances may vary among different assays in terms of sensitivity, specificity and calibration. Limited data are available about the comparability among LC-MS/ MS assays for serum steroids, mainly focusing on $\mathrm{T}$ and a few others. Though these data overall demonstrated the good inter-laboratory performance of LC-MS/MS (18, $43,44,45)$, no data are yet available on the majority of steroids included in modern large LC-MS/MS panels, and no certified materials or external quality assessment exist for many of them.

In conclusion, our study showed how excess weight and metabolic impairments frequently occurring in the 
general unmedicated adult population can significantly influence the circulating steroid profile and affect the RI estimation. Moreover, we provided age-specific RIs for seven steroids and unique RI for six other steroids to be used in the clinical management of several endocrine diseases. Future studies will be needed to test the efficacy of our RI in clinical settings, both for steroids with known diagnostic relevance and for those hormones and metabolites still neglected by the current clinical paradigms. Partial agreement with previous data was highlighted, thereby witnessing the urgent need for an overall harmonization in preanalytical and analytical aspects of steroid measurement that will definitely allow the harmonization of steroid reference values.

\section{Supplementary materials}

This is linked to the online version of the paper at https://doi.org/10.1530/ EJE-19-0928.

\section{Declaration of interest}

The authors declare that there is no conflict of interest that could be perceived as prejudicing the impartiality of this study.

\section{Funding}

The study was supported by the European Union (REPROBESITY, FPVII-223713), by the Emilia-Romagna Region - University Program 20072009 (g.a. PRUa1a-2007-006), by the Emilia-Romagna Region, Alessandro Liberati Young Researcher Grants (g.a. PRUA 1-2012-004) and by the Italian Ministry of Education, University and Research PRIN 2010-2011 (g.a. 2010C8ERKX).

\section{Author contribution statement}

M M measured study samples, performed the statistical analysis and wrote the manuscript. G D D, M B, A G, V V and C P performed cohort recruitment and examination. A F measured study samples. U P designed the population study and wrote the manuscript. F F designed the study and wrote the manuscript.

\section{Acknowledgements}

The authors thank Shimadzu for kindly providing the LC-MS/MS platform used to generate part of the data used in this study.

\section{References}

1 Ketha SS, Singh RJ \& Ketha H. Role of mass spectrometry in clinical endocrinology. Endocrinology and Metabolism Clinics of North America 201746 593-613. (https://doi.org/10.1016/j.ecl.2017.04.001)

2 Wudy SA, Schuler G, Sánchez-Guijo A \& Hartmann MF. The art of measuring steroids: principles and practice of current hormonal steroid analysis. Journal of Steroid Biochemistry and Molecular Biology 2018179 88-103. (https://doi.org/10.1016/j.jsbmb.2017.09.003)

3 La'ulu SL, Kalp KJ \& Straseski JA. How low can you go? Analytical performance of five automated testosterone immunoassays.
Clinical Biochemistry 201858 64-71. (https://doi.org/10.1016/j. clinbiochem.2018.05.008)

4 Yang DT, Owen WE, Ramsay CS, Xie H \& Roberts WL. Performance characteristics of eight estradiol immunoassays. American Journal of Clinical Pathology 2004122 332-337. (https://doi.org/10.1309/5N2R4HT4-GMOA-GPBY)

5 Yarrow JF, Beck DT, Conover CF, Beggs LA, Goldberger BA \& Borst SE. Invalidation of a commercially available human 5 $\alpha$-dihydrotestosterone immunoassay. Steroids 201378 1220-1225. (https://doi.org/10.1016/j.steroids.2013.08.013)

6 Schirpenbach C, Seiler L, Maser-Gluth C, Beuschlein F, Reincke M \& Bidlingmaier M. Automated chemiluminescenceimmunoassay for aldosterone during dynamic testing: comparison to radioimmunoassays with and without extraction steps. Clinical Chemistry 200652 1749-1755. (https://doi.org/10.1373/ clinchem.2006.068502)

7 Hawley JM, Owen LJ, Lockhart SJ, Monaghan PJ, Armston A, Chadwick CA, Wilshaw H, Freire M, Perry L \& Keevil BG. Serum cortisol: an up-to-date assessment of routine assay performance. Clinical Chemistry 201662 1220-1229. (https://doi.org/10.1373/ clinchem.2016.255034)

8 Fanelli F \& Di Dalmazi G. Serum steroid profiling by mass spectrometry in adrenocortical tumors: diagnostic implications. Current Opinion in Endocrinology, Diabetes, and Obesity 201926 160-165. (https://doi.org/10.1097/MED.0000000000000475)

9 Fanelli F, Baronio F, Ortolano R, Mezzullo M, Cassio A, Pagotto U $\&$ Balsamo A. Normative basal values of hormones and proteins of gonadal and adrenal functions from birth to adulthood. Sexual Development : Genetics, Molecular Biology, Evolution, Endocrinology, Embryology, and Pathology of Sex Determination and Differentiation 2018 12 50-94. (https://doi.org/10.1159/000486840)

10 Piché ME, Poirier P, Lemieux I \& Després JP. Overview of epidemiology and contribution of obesity and body fat distribution to cardiovascular disease: an update. Progress in Cardiovascular Diseases 201861 103-113. (https://doi.org/10.1016/j. pcad.2018.06.004)

11 Wu FC, Tajar A, Pye SR, Silman AJ, Finn JD, O’Neill TW, Bartfai G, Casanueva F, Forti G, Giwercman A et al. Hypothalamic-pituitary testicular axis disruptions in older men are differentially linked to age and modifiable risk factors: the European Male Aging Study. Journal of Clinical Endocrinology and Metabolism 200893 2737-2745. (https://doi.org/10.1210/jc.2007-1972)

12 Nieuwenhuizen AG \& Rutters F. The hypothalamic-pituitary-adrenalaxis in the regulation of energy balance. Physiology and Behavior 2008 94 169-177. (https://doi.org/10.1016/j.physbeh.2007.12.011)

13 Ceriotti F, Hinzmann R \& Panteghini M. Reference intervals: the way forward. Annals of Clinical Biochemistry 200946 8-17. (https://doi. org/10.1258/acb.2008.008170)

14 Ceglarek U, Werner M, Kortz L, Korner A, Kiess W, Thiery J \& Kratzsch J. Preclinical challenges in steroid analysis of human samples. Journal of Steroid Biochemistry and Molecular Biology 2010121 505-512. (https://doi.org/10.1016/j.jsbmb.2010.03.039)

15 Fanelli F, Belluomo I, Di Lallo VD, Cuomo G, De Iasio R, Baccini M, Casadio E, Casetta B, Vicennati V, Gambineri A et al. Serum steroid profiling by isotopic dilution-liquid chromatography-mass spectrometry: comparison with current immunoassays and reference intervals in healthy adults. Steroids 201176 244-253. (https://doi. org/10.1016/j.steroids.2010.11.005)

16 Matthews DR, Hosker JP, Rudenski AS, Naylor BA, Treacher DF $\&$ Turner RC. Homeostasis model assessment: insulin resistance and beta-cell function from fasting plasma glucose and insulin concentrations in man. Diabetologia 198528 412-419. (https://doi. org/10.1007/BF00280883)

17 Ingelsson E, Schaefer EJ, Contois JH, McNamara JR, Sullivan L, Keyes MJ, Pencina MJ, Schoonmaker C, Wilson PW, D'Agostino RB et al. Clinical utility of different lipid measures for prediction of 
coronary heart disease in men and women. JAMA 2007298 776-785. (https://doi.org/10.1001/jama.298.7.776)

18 Büttler RM, Martens F, Fanelli F, Pham HT, Kushnir MM, Janssen MJ, Owen L, Taylor AE, Soeborg T, Blankenstein MA et al. Comparison of 7 published LC-MS/MS methods for the simultaneous measurement of testosterone, androstenedione, and dehydroepiandrosterone in serum. Clinical Chemistry 201561 1475-1483. (https://doi. org/10.1373/clinchem.2015.242859)

19 Mezzullo M, Pelusi C, Fazzini A, Repaci A, Di Dalmazi G, Gambineri A, Pagotto U \& Fanelli F. Serum reference intervals for challenging sex and precursor steroids by liquid chromatography tandem mass spectrometry. Journal of Steroid Biochemistry and Molecular Biology 2019197 105538. (https://doi.org/10.1016/j. jsbmb.2019.105538)

20 Box GEP \& Cox DR. An analysis of transformations. Journal of the Royal Statistical Society: Series B 196426 211-243. (available at: https://www.jstor.org/stable/2984418) (https://doi. $\operatorname{org} / 10.1111 / \mathrm{j} .2517-6161.1964 . t b 00553 . x)$

21 Tukey JW. Exploratory Data Analysis. Addison-Wesley, 1977. (https:// doi.org/10.1002/bimj.4710230408)

22 Cohen J. Statistical Power Analysis for the Behavioral Sciences. Routledge, 1988. (https://doi.org/10.4324/9780203771587)

23 Clinical and Laboratory Standard Institute. Defining, Establishing, and Verifying Reference Intervals in the Clinical Laboratory; Approved Guideline, 3rd ed. CLSI document EP28-A3c: Wayne, PA: Clinical and Laboratory Standards Institute, 2008.

24 Royston P \& Wright EM. Method for estimating age-specic reference intervals (normal ranges) based on fractional polynomials and exponential transformation. Journal of the Royal Statistical Society: Series A 1998161 79-101. (https://doi.org/10.1111/1467985X.00091)

25 Perich C, Ricós C, Alvarez V, Biosca C, Boned B, Cava F, Doménech MV, Fernández-Calle $\mathrm{P}$, Fernández-Fernández $\mathrm{P}$, García-Lario JV et al. External quality assurance programs as a tool for verifying standardization of measurement procedures: pilot collaboration in Europe. Clinica Chimica Acta: International Journal of Clinical Chemistry 2014432 82-89. (https://doi.org/10.1016/j. cca.2013.11.005)

26 Vihma V, Naukkarinen J, Turpeinen U, Hämäläinen E, Kaprio J, Rissanen A, Heinonen S, Hakkarainen A, Lundbom J, Lundbom N et al. Metabolism of sex steroids is influenced by acquired adiposity - a study of young adult male monozygotic twin pairs. Journal of Steroid Biochemistry and Molecular Biology 2017172 98-105. (https:// doi.org/10.1016/i.jsbmb.2017.06.007)

27 Travison TG, Vesper HW, Orwoll E, Wu F, Kaufman JM, Wang Y, Lapauw B, Fiers T, Matsumoto AM \& Bhasin S. Harmonized reference ranges for circulating testosterone levels in men of four cohort studies in the United States and Europe. Journal of Clinical Endocrinology and Metabolism 2017102 1161-1173. (https://doi. org/10.1210/jc.2016-2935)

28 Sartorius G, Spasevska S, Idan A, Turner L, Forbes E, Zamojska A, Allan CA, Ly LP, Conway AJ, McLachlan RI et al. Serum testosterone, dihydrotestosterone and estradiol concentrations in older men self-reporting very good health: the healthy man study. Clinical Endocrinology 201277 755-763. (https://doi.org/10.1111/j.13652265.2012.04432.x)

29 Bae YJ, Zeidler R, Baber R, Vogel M, Wirkner K, Loeffler M, Ceglarek U, Kiess W, Körner A, Thiery J et al. Reference intervals of nine steroid hormones over the life-span analyzed by LC-MS/MS: effect of age, gender, puberty, and oral contraceptives. Journal of Steroid Biochemistry and Molecular Biology 2019193105409 (https:// doi.org/10.1016/j.jsbmb.2019.105409)

30 Van der Veen A, van Faassen M, de Jong WHA, van Beek AP, DijckBrouwer DAJ \& Kema IP. Development and validation of a LC-MS/MS method for the establishment of reference intervals and biological variation for five plasma steroid hormones. Clinical Biochemistry 2019 68 15-23. (https://doi.org/10.1016/j.clinbiochem.2019.03.013)

31 Kelsey TW, Li LQ, Mitchell RT, Whelan A, Anderson RA \& Wallace WH. A validated age-related normative model for male total testosterone shows increasing variance but no decline after age 40 years. PLoS ONE 20149 e109346. (https://doi.org/10.1371/journal. pone.0109346)

32 Wu A, Shi Z, Martin S, Vincent A, Heilbronn L \& Wittert G. Agerelated changes in estradiol and longitudinal associations with fat mass in men. PLoS ONE 201813 e0201912. (https://doi.org/10.1371/ journal.pone.0201912)

33 Rosmond R, Dallman MF \& Björntorp P. Stress-related cortisol secretion in men: relationships with abdominal obesity and endocrine, metabolic and hemodynamic abnormalities. Journal of Clinical Endocrinology and Metabolism 199883 1853-1859. (https:// doi.org/10.1210/jcem.83.6.4843)

34 Walker BR, Soderberg S, Lindahl B \& Olsson T. Independent effects of obesity and cortisol in predicting cardiovascular risk factors in men and women. Journal of Internal Medicine 2000247 198-204. (https:// doi.org/10.1046/j.1365-2796.2000.00609.x)

35 Cherradi N, Bideau M, Arnaudeau S, Demaurex N, James RW, Azhar S \& Capponi AM. Angiotensin II promotes selective uptake of high density lipoprotein cholesterol esters in bovine adrenal glomerulosa and human adrenocortical carcinoma cells through induction of scavenger receptor class B type I. Endocrinology 2001142 4540-4549. (https://doi.org/10.1210/endo.142.10.8412)

36 Eisenhofer G, Peitzsch M, Kaden D, Langton K, Pamporaki C, Masjkur J, Tsatsaronis G, Mangelis A, Williams TA, Reincke M et al. Reference intervals for plasma concentrations of adrenal steroids measured by LC-MS/MS: impact of gender, age, oral contraceptives, body mass index and blood pressure status. Clinica Chimica Acta: International Journal of Clinical Chemistry 2017470 115-124. (https:// doi.org/10.1016/j.cca.2017.05.002)

37 Damgaard-Olesen A, Johannsen TH, Holmboe SA, Søeborg T, Petersen JH, Andersson A, Aadahl M, Linneberg A \& Juul A. Reference ranges of 17-hydroxyprogesterone, DHEA, DHEAS, androstenedione, total and free testosterone determined by TurboFlow-LC-MS/MS and associations to health markers in 304 men. Clinica Chimica Acta: International Journal of Clinical Chemistry 2016454 82-88. (https:// doi.org/10.1016/j.cca.2015.12.042)

38 Kushnir MM, Rockwood AL, Bergquist J, Varshavsky M, Roberts WL Yue B, Bunker AM \& Meikle AW. High-sensitivity tandem mass spectrometry assay for serum estrone and estradiol. American Journal of Clinical Pathology 2008129 530-539. (https://doi.org/10.1309/ LC03BHQ5XJPJYEKG)

39 Kushnir MM, Rockwood AL, Roberts WL, Pattison EG, Owen WE, Bunker AM \& Meikle AW. Development and performance evaluation of a tandem mass spectrometry assay for 4 adrenal steroids. Clinical Chemistry 200652 1559-1567. (https://doi.org/10.1373/ clinchem.2006.068445)

40 Shiraishi S, Lee PW, Leung A, Goh VH, Swerdloff RS \& Wang C. Simultaneous measurement of serum testosterone and dihydrotestosterone by liquid chromatography-tandem mass spectrometry. Clinical Chemistry 200854 1855-1863. (https://doi. org/10.1373/clinchem.2008.103846)

41 Yeap BB, Knuiman MW, Divitini ML, Handelsman DJ, Beilby JP, Beilin J, McQuillan B \& Hung J. Differential associations of testosterone, dihydrotestosterone and oestradiol with physical, metabolic and health-related factors in community-dwelling men aged 17-97 years from the Busselton Health Survey. Clinical Endocrinology 201481 100-108. (https://doi.org/10.1111/ cen.12407)

42 Weckesser LJ, Plessow F, Pilhatsch M, Muehlhan M, Kirschbaum C $\&$ Miller R. Do venepuncture procedures induce cortisol responses? A review, study, and synthesis for stress research. 
Psychoneuroendocrinology 201446 88-99. (https://doi.org/10.1016/j. psyneuen.2014.04.012)

43 Thienpont LM, Van Uytfanghe K, Blincko S, Ramsay CS, Xie H, Doss RC, Keevil BG, Owen LJ, Rockwood AL, Kushnir MM et al. Stateof-the-art of serum testosterone measurement by isotope dilution-liquid chromatography-tandem mass spectrometry. Clinical Chemistry $2008 \mathbf{5 4}$ 1290-1297. (https://doi.org/10.1373/clinchem.2008.105841)

44 Vesper HW, Botelho JC, Vidal ML, Rahmani Y, Thienpont LM \& Caudill SP. High variability in serum estradiol measurements in men and women. Steroids 201482 7-13. (https://doi.org/10.1016/j. steroids.2013.12.005)

45 Büttler RM, Martens F, Ackermans MT, Davison AS, van Herwaarden AE, Kortz L, Krabbe JG, Lentjes EG, Syme C, Webster R et al. Comparison of eight routine unpublished LC-MS/MS methods for the simultaneous measurement of testosterone and androstenedione in serum. Clinica Chimica Acta: International Journal of Clinical Chemistry 2016454 112-118. (https://doi.org/10.1016/j. cca.2016.01.002)

Received 14 November 2019

Revised version received 20 February 2020

Accepted 2 March 2020 